\title{
La comunidad de Tuncarta, como potencial turístico del cantón Saraguro
}

\section{The community of Tuncarta, as tourism potential of the Saraguro canton}

\section{Pablo Antonio Abrigo Córdova}

Universidad Internacional del Ecuador, Ecuador

Autor para correspondencia: egtmaloja@gmail.com

Fecha de recepción: 26 de Abril de 2017 - Fecha de aceptación: 15 de Septiembre de 2017

Resumen: La Comunidad de Tuncarta, al no disponer de un inventario de atractivos turísticos elaborado, no cuenta con la herramienta básica para que la comunidad pueda desarrollar en forma definitiva, sustentable y sostenible en el ámbito turístico. A través de este proyecto, el cual ha sido ejecutado por los estudiantes de la Carrera de Ingeniería en Gestión Turística y Medio Ambiente de la UIDE - Loja conjuntamente con alumnos del Colegio de Bachillerato "Ñukanchik Kawsay" para Personas con Escolaridad Inconclusa perteneciente a la misma comunidad, se ha identificado, localizado y descrito los diferentes atractivos turísticos de las categorías sitios naturales y manifestaciones culturales existentes. Este estudio se lo realizó mediante una revisión bibliográfica y con la ayuda de trabajos anteriores elaborados en la comunidad, así como testimonios de personas estudiosas y conocedoras del tema, se realizó la recolección de datos y elaboración de fichas, estudio, análisis y reflexión de la realidad turística de Tuncarta. Con la elaboración del inventario turístico en el que se han descrito los atractivos en una forma técnica, resaltando sus características, calidad, apoyo y significado, de acuerdo al manual del Ministerio de Turismo existente, se dispone del instrumento fundamental para emprender en nuevos proyectos dirigidos a potenciar y revitalizar el turismo comunal que sea más integrador.

Palabras claves: atractivo turístico; potencial turístico; inventario turístico; categorías turísticas; turismo comunitario

\begin{abstract}
The community of Tuncarta, not having an inventory of tourist attractions drawn up, does not have the basic tool for the community to develop in a definitive, sustainable and sustainable way in the tourist area. Through this project, which has been carried out by the students of the UIDE - Loja Tourism and Environmental Management Engineering Course together with students of the "Ñukanchik Kawsay" Baccalaureate for Persons with Unfinished Schooling belonging to it Community, it has been identified, located and described the different tourist attractions of the categories natural sites and existing cultural manifestations. This study was carried out by means of a bibliographical review and with the help of previous works done in the community, as well as testimonies of people who are studious and knowledgeable about the subject, the data collection and preparation of fiches, study, analysis and reflection of the Tourist reality of Tuncarta. With the elaboration of the tourist inventory in which the attractions have been described in a technical way, highlighting their characteristics, quality, support and meaning, according to the manual of the existing Ministry of Tourism, the fundamental instrument is available to undertake new projects To promote and revitalize communal tourism that is more inclusive.
\end{abstract}

Key words: tourist attraction; tourist potential; tourist inventory; tourist categories; community tourism 


\section{Introducción}

\section{Desconocimiento de los principales atractivos turísticos en la comunidad de Tuncarta:}

El Proyecto de investigación se desarrolló en el sector de Tuncarta, Cantón Saraguro de la Provincia de Loja y nació a petición de la comunidad y como una alternativa de solución ante la problemática identificada como la falta de metodologías que permitan potencializar sus atractivos, ya sean naturales y culturales. Para ello fue necesario incorporar a los estudiantes de la Universidad Internacional del Ecuador Extensión Loja pertenecientes a la Carrera de Gestión Turística y Medio y del Colegio de Bachillerato "Ñukanchik Kawsay", cuyo beneficio fue para los habitantes del sector, ya que, ellos participaron activamente de este proceso de capacitación continua, por tal razón, se logró fortalecer su cultura, tradiciones e identidad nacional.

Esta investigación desde un inicio representó para la Carrera y la UIDE un fortalecimiento de su imagen por intermedio de los estudiantes al momento de realizar los aprendizajes.

Las actividades desplegadas en la presente investigación fortalecieron la relación directa entre los beneficios de la comunidad mediante la participación de los estudiantes y el buen vivir gestado en la comunidad (Abrigo, 2016).

La transmisión de las potencialidades identificadas técnicamente en el estudio a nivel nacional e internacional corresponderá a las autoridades de la comunidad y todos los involucrados en el turismo. Esperamos que el presente trabajo sirva como guía o base de investigaciones y de futuros proyectos que se puedan dar en este campo y que son necesarios para el desarrollo comunitario de los pueblos vulnerables.

En la presente investigación se propuso los siguientes objetivos:

- Evaluar los principales atractivos turísticos existentes en la comunidad de Tuncarta, cantón Saraguro, con la finalidad de evidenciar su potencial turístico.

- Desarrollar destrezas en la evaluación e identificación de los principales atractivos turísticos ambientales y culturales existentes en el sector.

- Inventariar los principales atractivos naturales y culturales del sector de Tuncarta para demostrar su potencia turístico.

- Socializar los resultados de la investigación a los beneficiarios directos e indirectos.

Hipótesis: El escaso conocimiento de los atractivos turísticos existentes en la comunidad de Tuncarta ha disminuido paulatinamente, motivado por diferentes factores económicos, sociales, lo cual puede ser mejorado si se identifican y jerarquizan los atractivos del sector. 
Los objetivos e hipótesis de la presente investigación están realacionados directamente, ya que se podrá inventariar los principales atractivos naturales y culturales en la comunidad de Tuncarta mediante la aplicación de las fichas del diagnóstico turístico (Ricaute, 2009).

Explore la Importancia del problema: Para la identificación, jerarquización y potenciación de los atractivos turísticos se propuso una metodología de fundamentación metodológica desde la perspectiva de la intervención comunitaria (Abrigo, 2016) que consintió en potenciar los atractivos turísticos emblemáticos en la comunidad de Tuncarta.

La identificación de los principales atractivos turísticos representan una actividad turística, natural y cultural, ósea las manifestaciones que caracterizaban al lugar, entre ellos tenemos, el tipo de gastronomía, siendo muy imprescindible destacar el tipo de cosecha que cada habitante realiza y que tiene que ver directamente con el tipo de comida única del lugar como por ejemplo la chicha de jora.

\section{Métodos}

Se ejecutó una investigación empírica - acción - participación, valiéndonos para ello de la observación directa y la aplicación de entrevistas y la realización de talleres y grupos focales. Además se efectuaron muestreos de toda la zona, requiriendo de varios integrantes de la comunidad y estudiantes de la UIDE y colegio.

Los métodos científicos que se utilizaron en la presente investigación fueron desglosados de la siguiente manera:

Histórico - Lógico: Se partió de la organización y análisis de la evolución histórica de los principales sucesos, son conceptos que aquí se tienen en cuenta (Desarrollo, atractivos turísticos naturales y culturales) para visualizar la trayectoria real de las causas y efectos que han generado el desconocimiento del potencial turístico en la comunidad de Tuncarta.

Las técnicas que se utilizaron en la presente investigación fueron desglosadas de la siguiente manera:

La observación: Visualizó la realidad del hecho a investigar.

La encuesta: Se la empleó para investigar masivamente determinados hechos o fenómenos que han propiciado la existencia de atractivos turísticos existentes en la comunidad, los cuales no han sido difundidos.

La entrevista a profundidad: Se la realizó a los representantes de organismos de apoyo social y a miembros de la comunidad de Tuncarta, a los cuales se los consideró como informantes claves, por ser hombres y mujeres de sabiduría y conocer las actividades del sector.

Fichas del Diagnóstico Turístico de Comunidades, Ficha de Caracterización de la Demanda y Ficha de Caracterización de Atractivos y Recursos Turísticos: Son formatos elaborados por el MINTUR, que permitieron obtener información turística de la comunidad. 


\section{Método de evaluación de atractivos utilizado por el M.I.N.T.U.R.}

La jerarquía se establece a partir de la suma de los valores asignados a cada factor, y en función de los puntos obtenidos se determina el rango jerárquico donde se ubica el atractivo.

\begin{tabular}{|c|c|c|c|c|}
\hline \multirow[t]{2}{*}{ VARIABLE } & & FACTOR & \multicolumn{2}{|c|}{ PUNTOS MÁXIMOS } \\
\hline & a) & Valor intrínseco & \multicolumn{2}{|l|}{15} \\
\hline \multirow[t]{5}{*}{ CALIDAD } & b) & Valor extrínseco & \multicolumn{2}{|l|}{15} \\
\hline & c) & Entorno & \multicolumn{2}{|l|}{10} \\
\hline & d) & Estado de Conservación & & 10 \\
\hline & & ( y/o organización) & \multicolumn{2}{|l|}{50} \\
\hline & a) & Acceso & \multicolumn{2}{|l|}{10} \\
\hline \multirow[t]{5}{*}{ APOYO } & b) & Servicios & \multicolumn{2}{|l|}{10} \\
\hline & c) & Asociación con otros & 5 & \\
\hline & & atractivos & \multicolumn{2}{|l|}{25} \\
\hline & a) & Local & 2 & \\
\hline & b) & Provincial & 4 & \\
\hline \multirow[t]{4}{*}{ SIGNIFICADO } & c) & Nacional & 7 & \\
\hline & d) & Internacional & \multicolumn{2}{|l|}{12} \\
\hline & & & \multicolumn{2}{|l|}{25} \\
\hline & TOTAL & & \multicolumn{2}{|l|}{100} \\
\hline
\end{tabular}

\begin{tabular}{|l|l|}
\hline RANGO & JERARQUÍAS \\
\hline 1 a 25 & I \\
\hline 26 a 50 & II \\
\hline 51 a 75 & III \\
\hline 76 a 100 & IV \\
\hline
\end{tabular}

Las variables propuestas en el estudio son las siguientes:

\section{Variables}

Variable dependiente: Identificación de los principales atractivos naturales y manifestaciones culturales de la comunidad de Tuncarta.

Variable independiente: Potencial turístico en la comunidad de Tuncarta.

Método de subsecciones etiquedos (Descripcion de los participantes): Los participantes del proyecto pertenecen a la comunidad de Tuncarta (480 habitantes) del cantón Saraguro de la provincia de Loja. Las principales actividades están destinadas a la agricultura, ganadería con animales menores y pocos práctican las artesanías en el sector. 
Población, muestra y medición: Para la población y muestra se consideró a moradores del sector de Tuncarta. La muestra utilizada fue la probabilística estratificada, ya que, la selección se llevó a cabo en forma mecánica y objetiva, fueron procedimientos aleatorios en

Cada estrato; en este caso se investigó a moradores de la comunidad de Tuncarta.

Las encuestas y entrevistas facilitaron la recolección de la información, la cual fue suficiente para verificar los objetivos planteados y la hipótesis; las cuales fueron analizadas e interpretadas por la muestra seleccionada.

Diseño de la investigación: El diseño de la investigación fue cualitativa, centrandose en la recopilación de información principalmente verbal y toma de datos por medio de fichas. Luego, la información obtenida fue analizada de una manera interpretativa, subjetiva, impresionista o incluso diagnóstica.

Características de los participantes: La participación de los moradores de la comunidad de Tuncarta fue fundamental para la realización de la investigación; ya que, sus conocimientos y sabiduría sirvió como referentes teóricos para completar la información; además fueron participes de las actividades de recopilación de información para inventariar los atractivos turísticos.

\section{Resultados}

\section{Desarrollar destrezas en la evaluación e identificación de los principales atractivos turísticos ambientales y culturales existentes en el sector:}

En la actualidad no se da importancia por parte de los profesionales del área ambiental y turística para el reconocimiento de lugares turísticos en la comunidad de Tuncarta. Es por ello que, resulta imprescindible para la Universidad Internacional del Ecuador Extensión Loja, apoyar a este tipo de proyectos, ya que, se pone de manifiesto la participación de sus alumnos, como gestores socioculturales (trasmisores de los conocimientos adquiridos en las aulas universitarias), con la comunidad que será beneficiada. Por este motivo se propuso una fundamentación metodológica desde la perspectiva de la intervención comunitaria enfocado en la interpretación turística de la comunidad de Tuncarta (Abrigo, 2016).

La finalidad de este proyecto fue desarrollar vínculos entre las instituciones implicadas en este proceso de trasmitir información técnica a información susceptible de aprender, además de facilitar a visitantes el recorrido por espacios seguros.

Antes de realizar las actividades planificadas en el proyecto se socializo con la comunidad la metodología a realizar en cada una de las etapas, para ello fue necesario vincular de manera directa a nuestros estudiantes y a los habitantes, para lo cual, se partió de las definiciones básicas para identificar los principales atractivos turísticos existentes y de esta manera desarrollar un turismo comunitario en el sector de Tuncarta, que a su vez no genere aspectos negativos o vaya contra la naturaleza que existe en el sector. 
Con esta información básica se dio inicio al mismo; implicando èste tipo de proyecto una actividad turística, cultural y natural. Se fue compartiendo conocimientos con los estudiantes del colegio y comunidad, con los cuales se llevó a cabo el proyecto, siendo necesario la identificación de los atractivos del lugar sean éstos de aspecto cultural; es decir, las manifestaciones que caracterizaban al lugar y el tipo de gastronomía, siendo muy imprescindible destacar el tipo de cosecha que cada habitante realiza tiene que ver directamente con el tipo de comida única del lugar como por ejemplo la chicha de jora. Podemos mencionar que el arte culinario de esta comunidad es empírico, ya que, se transmite de generación en generación, perdurando de esta manera el folklore y las costumbres de esta zona de nuestra provincia.

Podemos encontrar platos típicos de sencilla elaboración que van desde una colada de zambo a otros más elaborados; cabe mencionar que toda la materia prima con la que elaboran la comida típica es sembrada y cosecha da de forma totalmente orgánica.

Otra manifestación que se da en él es la fabricación de sombreros que destacan al sector como productor de mismo, no solo en ese aspecto cultural sino también natural, ya que viven alrededor de pequeñas montañas donde se pueden realizar actividades de caminatas pero sin antes incluir un buen sendero. Todas estas actividades identificadas fueron reconocidas por los jóvenes que facilitaron la información para levantar las fichas, las cuales ayudaron a elaborar un inventario de atractivos del lugar.

Inventariar los principales atractivos naturales y culturales del sector de Tuncarta para demostrar su potencia turística: a continuación damos a conocer el inventario de atractivos naturales y manifestaciones culturales de la comunidad de Tuncarta

Tabla 1. Atractivos turísticos y su potencial turístico en la comunidad de Tuncarta,

\begin{tabular}{|c|c|c|c|c|c|c|}
\hline $\begin{array}{l}\mathbf{N} \\
\mathbf{r} \\
\mathbf{0 .}\end{array}$ & Categoría & Nombre & Tipo & Subtipo & $\begin{array}{l}\text { Puntaje } \\
\text { de } \\
\text { Atractiv } \\
\text { o }\end{array}$ & $\begin{array}{l}\text { Jer } \\
\text { arq } \\
\text { uía }\end{array}$ \\
\hline & \multicolumn{6}{|c|}{ Sitios Naturales } \\
\hline 1 & $\begin{array}{l}\text { Sitios } \\
\text { Naturales }\end{array}$ & Paisaje de Tuncarta & $\begin{array}{l}\text { Ambientes } \\
\text { Lacustres }\end{array}$ & Paisaje & 43 & II \\
\hline 2 & $\begin{array}{l}\text { Sitios } \\
\text { Naturales }\end{array}$ & Lago de Tuncarta & $\begin{array}{l}\text { Ambientes } \\
\text { Lacustres }\end{array}$ & Lago & 25 & I \\
\hline 3 & $\begin{array}{l}\text { Sitios } \\
\text { Naturales } \\
\text { Manifestac }\end{array}$ & $\begin{array}{l}\text { Bosque de Tuncarta } \\
\text { ones Culturales }\end{array}$ & Bosque & Montano bajo occidental & 32 & $\begin{array}{l}\text { II } \\
\text { II }\end{array}$ \\
\hline 4 & $\begin{array}{l}\text { Manifestac } \\
\text { iones } \\
\text { Culturales }\end{array}$ & Chica de jroa & Etmografía & $\begin{array}{l}\text { Comidas típicas y } \\
\text { bebidas }\end{array}$ & 29 & II \\
\hline 5 & $\begin{array}{l}\text { Manifestac } \\
\text { iones } \\
\text { Culturales }\end{array}$ & Colada de Zapallo & Etmografía & $\begin{array}{l}\text { Comidas típicas y } \\
\text { bebidas }\end{array}$ & 30 & II \\
\hline 6 & $\begin{array}{l}\text { Manifestac } \\
\text { iones } \\
\text { Culturales }\end{array}$ & Ají de pepa de zambo & Etmografía & $\begin{array}{l}\text { Comidas típicas y } \\
\text { bebidas }\end{array}$ & 29 & II \\
\hline
\end{tabular}




\begin{tabular}{|c|c|c|c|c|c|c|}
\hline 7 & $\begin{array}{l}\text { Manifestac } \\
\text { iones } \\
\text { Culturales }\end{array}$ & Cebada picada con queso & Etmografía & $\begin{array}{l}\text { Comidas típicas y } \\
\text { bebidas }\end{array}$ & 29 & II \\
\hline 8 & $\begin{array}{l}\text { Manifestac } \\
\text { iones } \\
\text { Culturales }\end{array}$ & Feria de Remates & Etmografía & Ferias y Mercados & 24 & I \\
\hline 9 & $\begin{array}{l}\text { Manifestac } \\
\text { iones } \\
\text { Culturales }\end{array}$ & Feria de Artesanías & Etmografía & Ferias y Mercados & 23 & I \\
\hline $\begin{array}{l}1 \\
0\end{array}$ & $\begin{array}{l}\text { Manifestac } \\
\text { iones } \\
\text { Culturales }\end{array}$ & Elaboración de tejidos & Etmografía & Artesanías & 44 & II \\
\hline $\begin{array}{l}1 \\
1\end{array}$ & $\begin{array}{l}\text { Manifestac } \\
\text { iones } \\
\text { Culturales }\end{array}$ & $\begin{array}{l}\text { Elaboración de Sombreros de } \\
\text { Lana }\end{array}$ & Etmografía & Artesanías & 45 & II \\
\hline $\begin{array}{l}1 \\
2\end{array}$ & $\begin{array}{l}\text { Manifestac } \\
\text { iones } \\
\text { Culturales }\end{array}$ & Elaboración de Vetas & Etmografía & Artesanías & 44 & II \\
\hline $\begin{array}{l}1 \\
3\end{array}$ & $\begin{array}{l}\text { Manifestac } \\
\text { iones } \\
\text { Culturales }\end{array}$ & Elaboración de collares & Etmografía & Artesanías & 34 & II \\
\hline $\begin{array}{l}1 \\
4\end{array}$ & $\begin{array}{l}\text { Manifestac } \\
\text { iones } \\
\text { Culturales }\end{array}$ & Virgen del Cisne & Etmografía & $\begin{array}{l}\text { Manifestaciones } \\
\text { religiosas, tradicionales y } \\
\text { creencias populares }\end{array}$ & 28 & II \\
\hline 1 & $\begin{array}{l}\text { Manifestac } \\
\text { iones } \\
\text { Culturales }\end{array}$ & Semana Santa & Etmografía & $\begin{array}{l}\text { Manifestaciones } \\
\text { religiosas, tradicionales y } \\
\text { creencias populares }\end{array}$ & 28 & II \\
\hline $\begin{array}{l}1 \\
6\end{array}$ & $\begin{array}{l}\text { Manifestac } \\
\text { iones } \\
\text { Culturales }\end{array}$ & San Isidro & Etmografía & $\begin{array}{l}\text { Manifestaciones } \\
\text { religiosas, tradicionales y } \\
\text { creencias populares }\end{array}$ & 28 & II \\
\hline $\begin{array}{l}1 \\
7\end{array}$ & $\begin{array}{l}\text { Manifestac } \\
\text { iones } \\
\text { Culturales }\end{array}$ & Día de los difuntos & Etmografía & $\begin{array}{l}\text { Manifestaciones } \\
\text { religiosas, tradicionales y } \\
\text { creencias populares }\end{array}$ & 28 & II \\
\hline $\begin{array}{l}1 \\
8\end{array}$ & $\begin{array}{l}\text { Manifestac } \\
\text { iones } \\
\text { Culturales }\end{array}$ & $\begin{array}{l}\text { Creencia popular "persona } \\
\text { agonizando" }\end{array}$ & Etmografía & $\begin{array}{l}\text { Manifestaciones } \\
\text { religiosas, tradicionales y } \\
\text { creencias populares }\end{array}$ & 24 & I \\
\hline $\begin{array}{l}1 \\
9\end{array}$ & $\begin{array}{l}\text { Manifestac } \\
\text { iones } \\
\text { Culturales }\end{array}$ & $\begin{array}{l}\text { Creencia popular "siembra por } \\
\text { una mujer embarazada" }\end{array}$ & Etmografía & $\begin{array}{l}\text { Manifestaciones } \\
\text { religiosas, tradicionales y } \\
\text { creencias populares }\end{array}$ & 24 & I \\
\hline $\begin{array}{l}2 \\
0\end{array}$ & $\begin{array}{l}\text { Manifestac } \\
\text { iones } \\
\text { Culturales }\end{array}$ & Creencia popular "el sapo" & Etmografía & $\begin{array}{l}\text { Manifestaciones } \\
\text { religiosas, tradicionales y } \\
\text { creencias populares }\end{array}$ & 25 & I \\
\hline $\begin{array}{l}2 \\
1\end{array}$ & $\begin{array}{l}\text { Manifestac } \\
\text { iones } \\
\text { Culturales }\end{array}$ & Creencia tradicional "La Araña" & Etmografía & $\begin{array}{l}\text { Manifestaciones } \\
\text { religiosas, tradicionales y } \\
\text { creencias populares }\end{array}$ & 24 & I \\
\hline $\begin{array}{l}2 \\
2\end{array}$ & $\begin{array}{l}\text { Manifestac } \\
\text { iones } \\
\text { Culturales }\end{array}$ & Grupo Étnico "Los Saraguros" & Etmografía & Grupo Étnico & 39 & II \\
\hline $\begin{array}{l}2 \\
3\end{array}$ & $\begin{array}{l}\text { Manifestac } \\
\text { iones } \\
\text { Culturales }\end{array}$ & Eaboración del Zamarro & Etmografía & Indumentaria & 44 & II \\
\hline $\begin{array}{l}2 \\
4\end{array}$ & $\begin{array}{l}\text { Manifestac } \\
\text { iones } \\
\text { Culturales }\end{array}$ & Fiesta educativa "Navidad" & $\begin{array}{l}\text { Acontecimi } \\
\text { entos } \\
\text { programado } \\
\text { s }\end{array}$ & Fiestas & 25 & I \\
\hline
\end{tabular}




\begin{tabular}{|c|c|c|c|c|c|c|}
\hline $\begin{array}{l}2 \\
5\end{array}$ & $\begin{array}{l}\text { Manifestac } \\
\text { iones } \\
\text { Culturales }\end{array}$ & $\begin{array}{l}\text { Aniversario del colegio de } \\
\text { Tuncarta }\end{array}$ & $\begin{array}{l}\text { Acontecimi } \\
\text { entos } \\
\text { programado } \\
\text { s }\end{array}$ & Fiestas & 24 & I \\
\hline $\begin{array}{l}2 \\
6\end{array}$ & $\begin{array}{l}\text { Manifestac } \\
\text { iones } \\
\text { Culturales }\end{array}$ & $\begin{array}{l}\text { Arquitectura religiosa loma de } \\
\text { cruz }\end{array}$ & Históricas & Arquitectura religiosa & 20 & I \\
\hline $\begin{array}{l}2 \\
7\end{array}$ & $\begin{array}{l}\text { Manifestac } \\
\text { iones } \\
\text { Culturales }\end{array}$ & $\begin{array}{l}\text { Arquitectura religiosa Capilla de } \\
\text { Tuncarta }\end{array}$ & Históricas & Arquitectura religiosa & 21 & I \\
\hline $\begin{array}{l}2 \\
8\end{array}$ & $\begin{array}{l}\text { Manifestac } \\
\text { iones } \\
\text { Culturales }\end{array}$ & $\begin{array}{l}\text { Arquitectura de las casas } \\
\text { antiguas (adobe y bareque) }\end{array}$ & Históricas & Arquitectura Civil & 19 & I \\
\hline $\begin{array}{l}2 \\
9\end{array}$ & $\begin{array}{l}\text { Manifestac } \\
\text { iones } \\
\text { Culturales }\end{array}$ & $\begin{array}{l}\text { Arquitectura de las casas } \\
\text { modernas (cemento, ladrillo, } \\
\text { bloque, teja, zinc, madera) }\end{array}$ & Históricas & Arquitectura Civil & 19 & I \\
\hline
\end{tabular}

\section{Principales atractivos turísticos de la comunidad de Tuncarta}

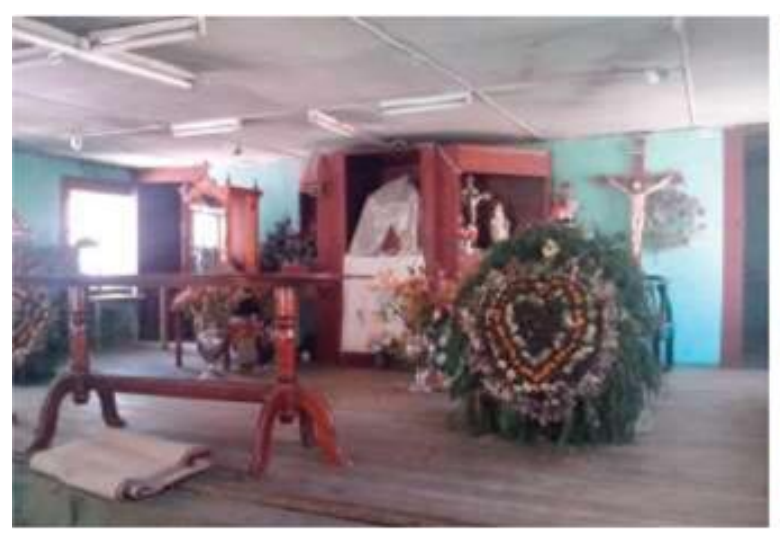

Figura 1. Capilla de Tuncarta

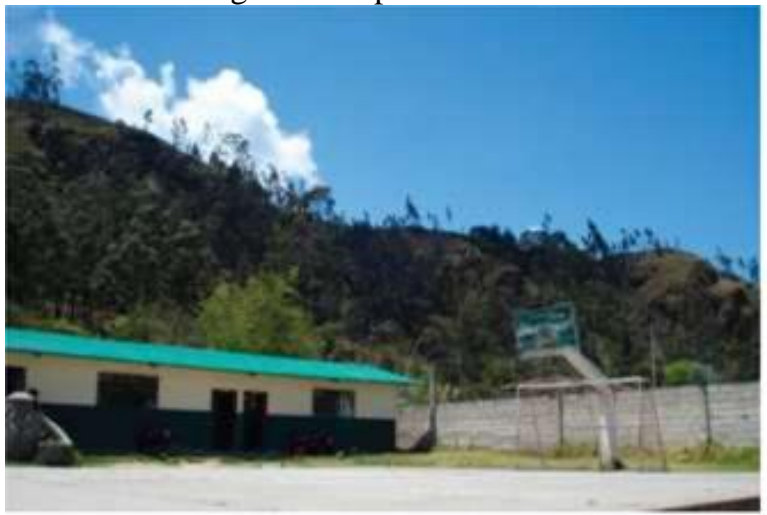

Figura 3. Colegio de Bachillerato Ñukanchik Kawsay

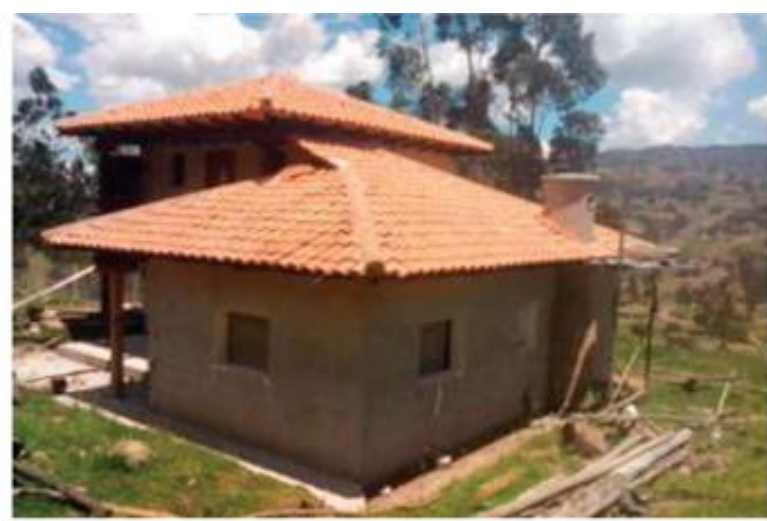

Figura 2. Casas elaboradas de Adobe y bareque

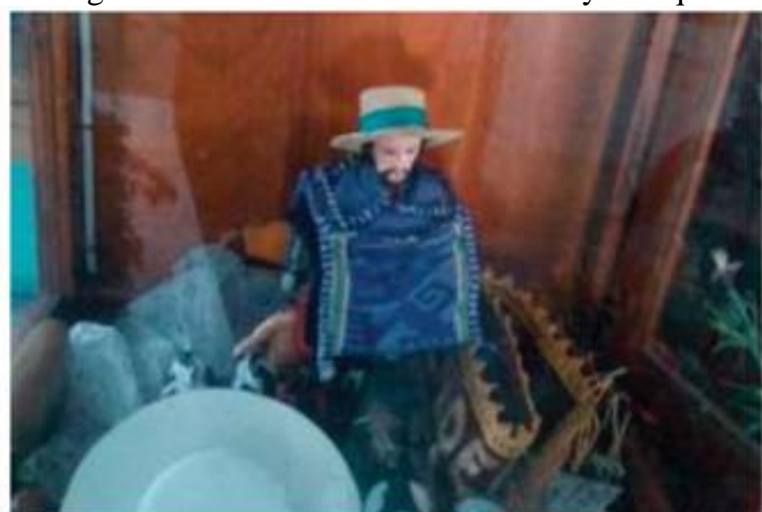

Figura 4. Manifestación religiosa San Isidro 


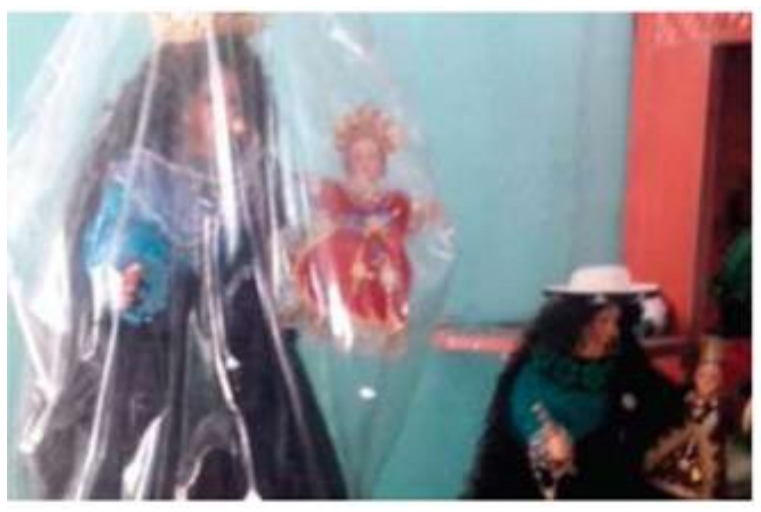

Figura 5. Manifestación religiosa y tradicional virgen del Cisne

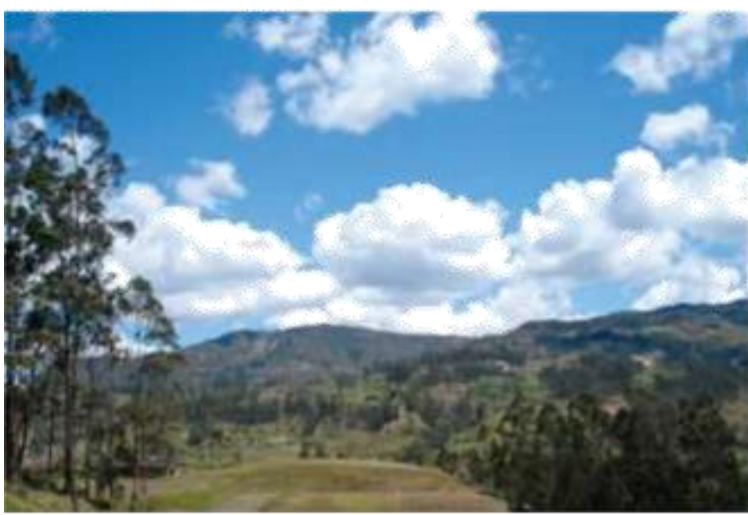

Figura 7. Bosque de Tuncarta

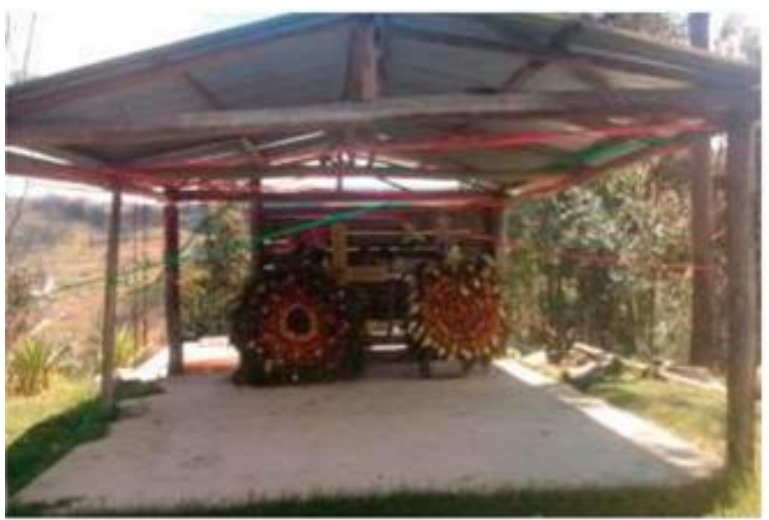

Figura 9. Manifestación religiosa. Loma de la Cruz

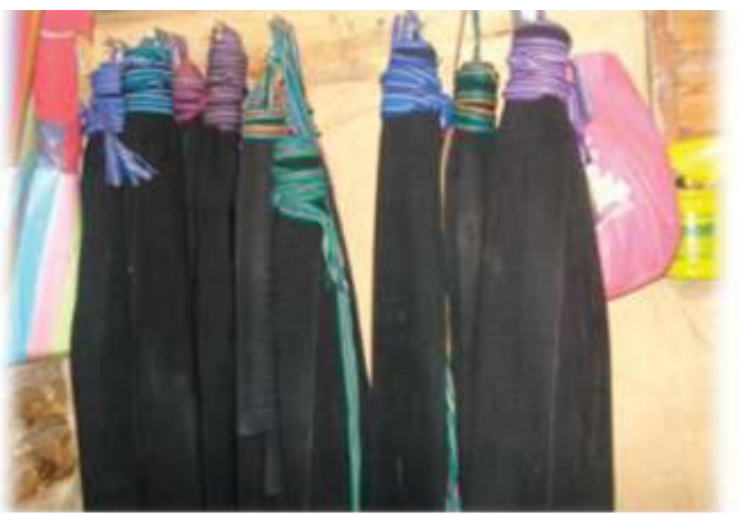

Figura 6. Elaboración de tejidos, reboso

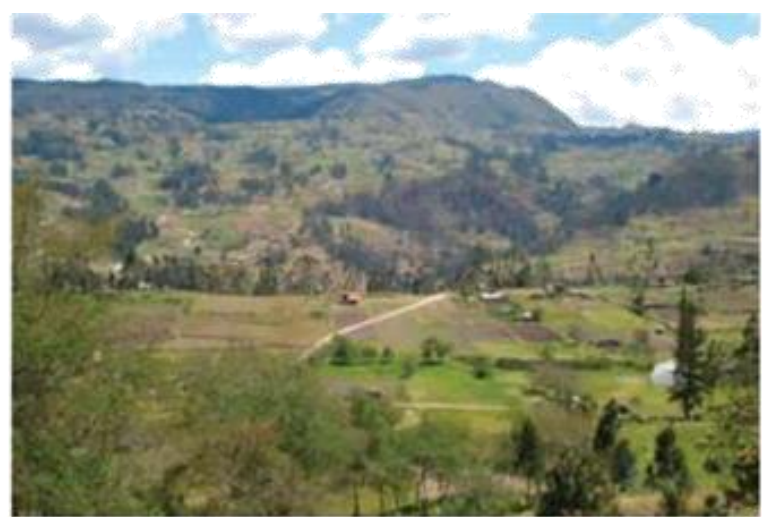

Figura 8. Paisaje de Tuncarta

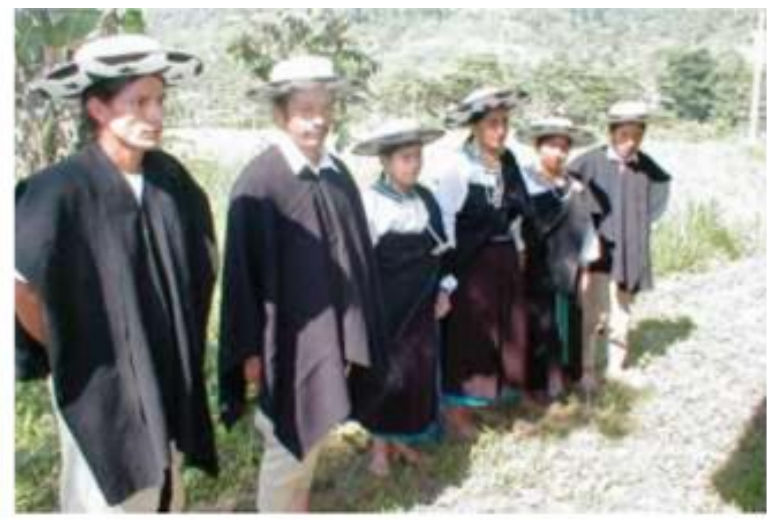

Figura 10. Grupo étnico. Los saraguros.

(Fuente: Wilson Armijos) 


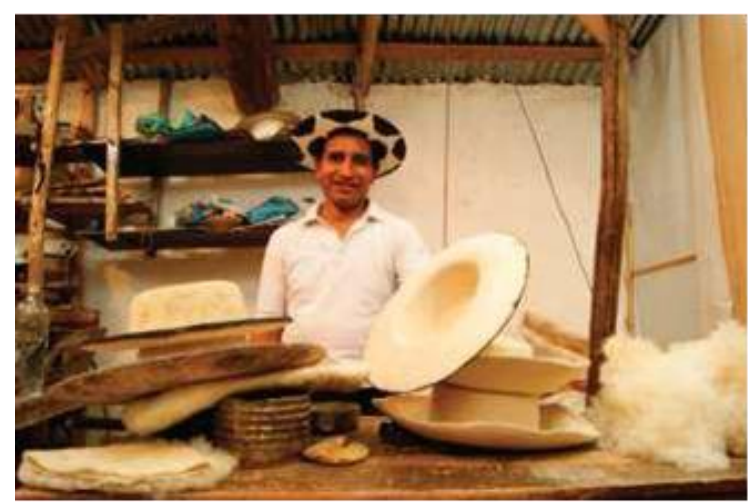

Figura 11. Elaboración de sombreros. Propietario Francisco Sarango

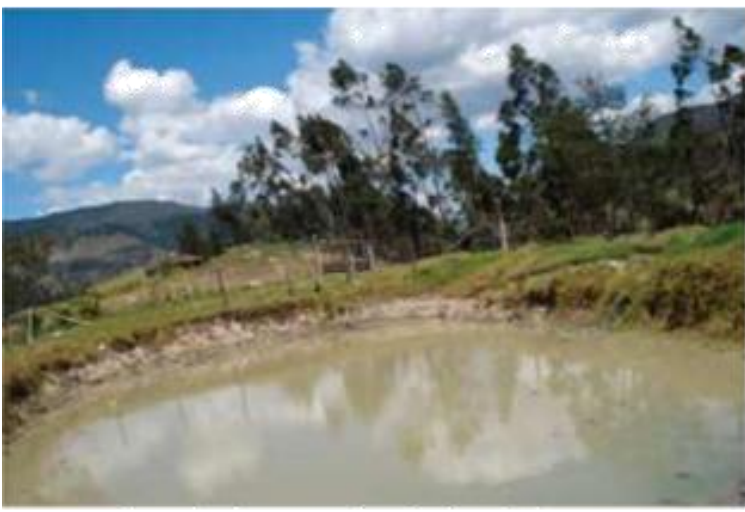

Figura 13. Lago de Tuncarta

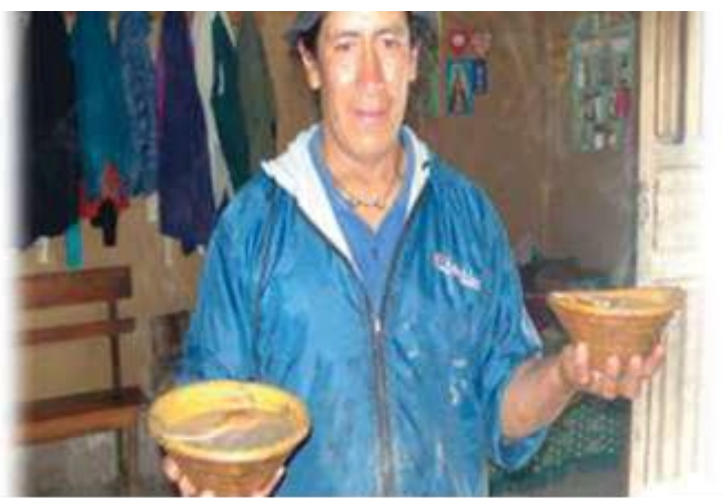

Figura 12. Plato típico de cebada

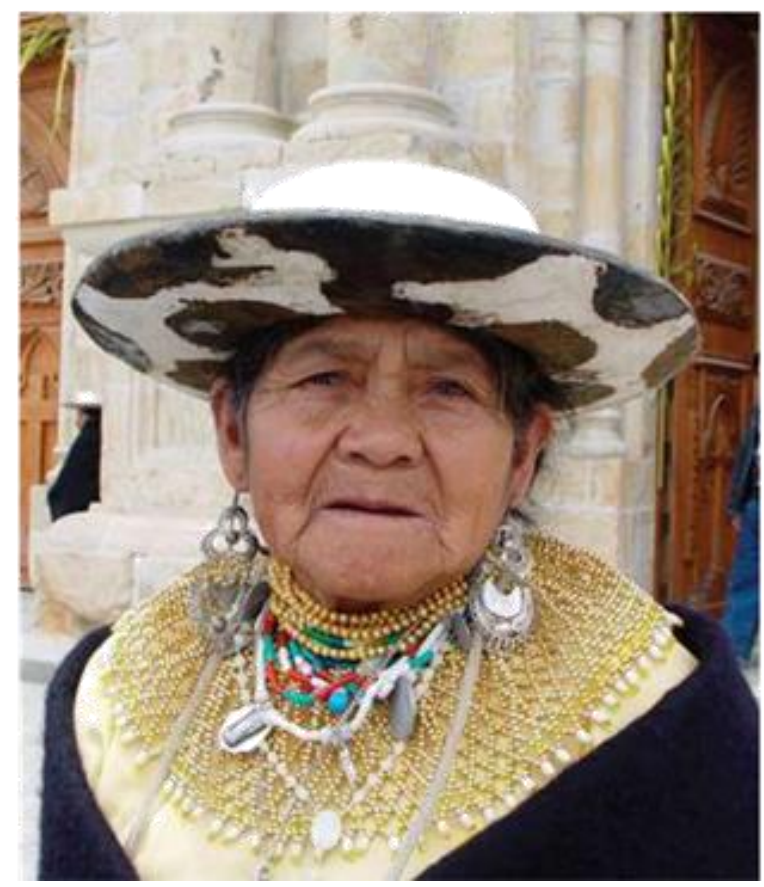

Figura 14. Elaboración de collares

Para potencializar los atractivos turísticos en la comunidad de Tuncarta, se propuso dos paquetes turísticos, los cuales fueron elaborados por los estudiantes de la CGTMA - UIDE Loja.

\section{Maravillas del austro}

\section{Cotización en base a diez adultos}

La práctica del turismo comunitario brinda la esperanza de una mejor calidad de vida a nivel familiar y comunitario, conservando los recursos naturales y revalorizando la cultura de las comunidades.

Duración: 4 días 
Altitud: 2500 m.s.n.m

Dificultad: Fácil - moderada

Carácter: Historia, naturaleza, cultura y arqueología

\section{El programa incluye}

Día: 1

$>$ Traslado Cuenca - Saraguro - Tuncarta

$>$ Desayuno

$>$ Llega a Saraguro

$>$ Visita a los telares

$>$ Almuerzo

Visita a la comunidad Tuncarta

$>$ Retorno a Cuenca

$>$ Alojamiento en Cuenca

$>$ Cena

Día: 2

$>$ Desayuno

$>$ City Tour en Cuenca

$>$ Almuerzo

$>$ Cena

$>$ Alojamiento

Día: 3

$>$ Desayuno

$>$ Visita al Complejo Arqueológico de Inga Pirca

$>$ Almuerzo

$>$ Visita a la cara del inca y la iglesia de la Virgen del Rocío

$>$ Paseo panorámico, mirador de Turi

$>$ Cena

$>$ Alojamiento

Día: 4

$>$ Desayuno

$>$ Parque Nacional del Cajas

$>$ Almuerzos

$>$ Traslado al Aeropuerto

Se trata de un concepto totalmente diferente y novedoso de hacer turismo desde un punto de vista intercultural. Familias indígenas comparten sus viviendas con los turistas o visitantes y permiten conocer a fondo sus costumbres, su forma de vida, sus conocimientos 
Por sólo: \$ $260 \quad$ USD = Alojamiento +3 comidas y actividades con la familia

Incluye

$>$ Transporte con seguro SOAT

$>$ Guía coordinador

$>$ Botella con agua

$>$ Entradas

$>$ Solo es cotización de precios puede variar

\section{Disfruta de las tradiciones}

\section{Cotización en base a 10 adultos}

Duración: 1 día

Altitud: 2500 m.s.n.m

Dificultad: fácil - moderada

Carácter: Historia, naturaleza, cultura y arqueología

El programa incluye

$>$ Trasporte Loja - Saraguro - Loja

$>$ Desayuno

$>$ Llegada a Saraguro

$>$ Visita al Baño del Inca

$>$ Visita a los Telares

$>$ Almuerzo

$>$ Visita a la Comunidad de Tuncarta y recorrido por sus principales atractivos

$>$ Retorno a Loja

$>$ Cena

\section{Precio por persona USD 30.00}

\section{Incluye}

$>$ Guía coordinador

$>$ Alimentación completa

$>$ Botella con agua

$>$ Entradas

Solo es cotización los precios pueden variar

$>$ Transporte con seguro SOAT 
Socializar los resultados de la investigación a los beneficiarios directos e indirectos: Esta actividad se desarrolló en el Salón Social de la Escuela Lago de San Pablo ubicada en el sector de Tuncarta, para ello asistieron estudiantes de ambas instituciones y comunidad en general. En este evento se dio a conocer los principales atractivos turísticos existentes en la comunidad de Tuncarta.

\section{Discusión}

El enfoque aplicado en la investigación fue el mixto; es decir, se refiere a la metodología cualitativa que se empleó, representando al tamaño muestral necesario en función de encuestas, observaciones y entrevistas aplicadas; las cuales describieron la cotidianidad, aspectos socioculturales, situaciones problemáticas y los significados de la vida de las personas de la comunidad de Tuncarta (Abrigo, 2016).

La otra metodología que se utilizó fue, la cuantitativa, la cual se fundamentó en la búsqueda de las causas que generaron el desconocimiento de los atractivos turísticos, persiguiendo la razón y la explicación; permitiéndose dar cuenta de contextos, estructuras y alcance social de problemáticas que fueron tratadas en lo particular.

En el contexto social el proyecto aspira a ser uno de los medios más eficaces para generar la participación ciudadana dentro de la actividad turística, impulsando la creación de empleo y el desarrollo económico en la comunidad local.

También, se busca promover el respeto al medio ambiente por medio de la interpretación ambiental, acción que procura contribuir la conservación de los ecosistemas y la concientización de los visitantes.

Esta propuesta está dirigida a todos los segmentos de la población incluyendo la tercera edad y personas con capacidades especiales. En términos de género la recreación no es excluyente, es un vínculo para la cohesión social, familiar y laboral.

La Comunidad en mención, al no disponer de un inventario de atractivos turísticos elaborado, no cuenta con la herramienta básica para que la comunidad pueda despegar en forma definitiva, sustentable y sostenible en el ámbito turístico.

A través de este proyecto, el cual ha sido ejecutado por los estudiantes de la Carrera de Ingeniería en Gestión Turística y Medio Ambiente conjuntamente con alumnos del Colegio de Bachillerato "Ñukanchik Kawsay" para Personas con Escolaridad Inconclusa perteneciente a la misma comunidad, cuyo beneficio fue para los 480 habitantes del sector, las actividades lograron fortalecer su cultura, tradiciones e identidad nacional. El Diseño propuesto, nació como resultado del estudio de la matriz para el diagnóstico participativo aplicado (Abrigo, 2016).

Este estudio se lo realizó mediante una revisión bibliográfica y con la ayuda de trabajos anteriores elaborados en la comunidad, así como testimonios de personas estudiosas y conocedoras del tema, se realizó la recolección de datos y elaboración de fichas, estudio, análisis y reflexión de la realidad turística de Tuncarta. 
El impacto del proyecto en la comunidad ha sido muy relevante, ya que permitió potencializar turísticamente a la comunidad, mediante la elaboración del inventario turístico en el que se han descrito los atractivos en una forma técnica, resaltando sus características calidad, apoyo y significado, de acuerdo al manual del Ministerio de Turismo existente, se dispone del instrumento fundamental para emprender en nuevos proyectos dirigidos a potenciar y revitalizar el turismo comunal que sea más integrador. Se consideran dos paquetes turísticos los cuales se los difundió para potencializar turísticamente la comunidad de Tuncarta. La transmisión de las potencialidades identificadas técnicamente en el estudio a nivel nacional e internacional corresponderá a las autoridades de la comunidad y todos los involucrados en el turismo.

\section{Conclusiones}

La Comunidad de Tuncarta dispone de atractivos turísticos, como sitios naturales y manifestaciones culturales, convirtiéndose en un lugar con gran potencial, para desarrollar el turismo provincial, nacional e internacional.

La gran mayoría de los atractivos han sido levantados mediante la aplicación de fichas y de acuerdo a la jerarquización se observa que pertenecen a la categoría I y II.

El turismo en la Comunidad se ha constituido en una actividad que generaría ingresos económicos a los diferentes sectores.

La Comunidad no dispone de un inventario turístico, técnicamente elaborado de acuerdo a las especificaciones del Ministerio de Turismo.

Un inventario de atractivos turísticos, es el instrumento básico y elemental para emprender en diferentes estudios y proyectos que ayudarán a potenciar el turismo en la comunidad.

En este proyecto se compiló información turística y ambiental para potencializarlos y así aumentar sus ingresos económicos de sus familias.

Además por medio de la interpretación se deberá enseñar no solo al turista sino también a la comunidad y principalmente a los niños a cuidar su espacio tanto cultural como natural.

Con toda esta información las familias junto con la comunidad pueden pensar en iniciar en proyectos turísticos comunitarios conjuntamente con el agroturismo, con un enfoque sustentable.

Los resultados obtenidos para la comunidad mediante la ejecución del proyecto de señalética fueron muy satisfactorios, de acuerdo a los resultados obtenidos de las encuestas.

La ejecución de la propuesta genera empleo permanente lo que se transforma en un beneficio social para la colectividad, contribuyendo al fortalecimiento del desarrollo integral del ser humano. 
Mediante la identificación de los principales atractivos turísticos existentes se desarrollará turismo comunitario en el sector de Tuncarta.

La Comunidad de Tuncarta dispone de los diferentes atractivos turísticos, como sitios naturales y manifestaciones culturales.

Nuestro agradecimiento imperecedero a los docentes y alumnos de la Carrera en Gestión Turística y Medio Ambiente de la Universidad Internacional del Ecuador extensión Loja y del Colegio de Bachillerato NukanchikKawsay (Nuestra Vida) PEI, quienes con su sacrificio y entrega mostrado cada semana permitieron que este trabajo llegué a su feliz término.

De nosotros, especial testimonio de reconocimiento a la comunidad de Tuncarta, especialmente a los Directivos del Colegio, por la colaboración al brindarnos las facilidades para el desarrollo de la investigación en mención y a todos que de alguna manera impulsaron para que se tenga el éxito deseado.

\section{Bibliografía}

Abrigo, P. A. A. (2016). Identificación de Necesidades para Proponer el Desarrollo Turístico en la Comunidad de Tuncarta, del Cantón Saraguro de la Provincia de Loja. INNOVA Research Journal, 1(6).

Abrigo P. (2008). Proyecto de ampliación al servicio de Bachillerato en el Colegio Agropecuario Nukanchik Kawsay. Saraguro - Ecuador

Bullón, R. 1993. Actividades Turísticas Recreacionales. México. Edit. Trillas.

Calero J. (2000). Investigación cualitativa y cuantitativa. Problemas no resueltos en los debates actuales. Rev. Cubana Endocrinol; 11 (3): 192-8.

Garcia P. (Ministerio de Turismo 2006). Guía de Inventarios para implementación de Productos Turísticos.

Herrera, L. N., \& Guzman, P. V. (2013). Plan de desarrollo local para la comunidad Vicente León, parroquia Toacaso, cantón Latacunga, provincia de Cotopaxi. Tésis Eco. Latacunga, Ec., Universidad Central del Ecuador, Facultad de Ciencias Económicas. -Jeans, J. (2004). Marketing Estratégico. Edit. Mc. Graw Hill. Madriz.

Marchioni, M., (1999). C Comunidad, participación y desarrollo. $\quad$ Madrid., Popular S.A.

Martínez, M. (2011). Desarrollo Sociocultural comunitario. Universidad Central "Martha Abrew" de la Villas, Santa Clara - Cuba.

Ricaurte Quijano, C. V. (2009). Manual para el diagnóstico turístico local. 
Secretaria Nacional de Planificación y Desarrollo. (2013)., Plan Nacional delñ Buien vivir 2013 2017. Quito - Ecuador.

Verdejo, M. E., \& Hidalgo, E. J. (2003). Diagnóstico rural participativo: Una guía práctica.

Centro Cultural Poveda, Proyecto Comunicación y Didáctica. 